\title{
VALORACIÓN DE LOS FACTORES QUE CONDICIONAN LA ADQUISICIÓN DE HÁBITOS ALIMENTARIOS SALUDABLES EN PERSONAS CON TRASTORNO MENTAL GRAVE (TMG): APROXIMACIÓN CUALITATIVA DESDE UNA PERSPECTIVA EN PRIMERA PERSONA Y DE LOS PROFESIONALES
}

\author{
Mireia Vilamala-Orra1, Cristina Vaqué-Crusellas2 y Ruben del Rio Saéz1 \\ ${ }^{1}$ Fundació Centre Mèdic Psicopedagògic d'Osona y Universidad de Vic - Universidad Central de Cataluña, España. \\ mvilamala@osonament.cat \\ ${ }^{2}$ Universidad de Vic - Universidad Central de Cataluña, España. cristina.vaque@uvic.cat
}

\begin{abstract}
Resumen. Introducción: La adopción de dietas de baja calidad nutricional se relaciona con la aparición de alteraciones metabólicas, factor que contribuye a la elevada tasa de comorbilidades entre las personas con TMG. Conocer los factores condicionantes permite desarrollar programas de educación alimentaria adecuados a las necesidades de la persona; Objetivos: Explorar los factores que condicionan la adopción y mantenimiento de los hábitos alimentarios, e identificar las estrategias más eficientes para el cambio de hábitos; Métodos: Estudio descriptivo mediante la recogida de datos y análisis cualitativo. Los datos se obtuvieron de la realización de 6 grupos de discusión. Se procedió a un análisis temático del corpus textual; Resultados: Participaron 23 personas vinculadas a los servicios de salud mental y 30 profesionales de los distintos ámbitos de atención. Se desprenden 6 temas principales; Conclusiones: Profesionales y usuarios coinciden en la necesidad de promocionar la alimentación saludable. Conocer la predisposición y los condicionantes para adoptar un cambio de conducta es fundamental para el diseño de intervenciones eficientes.
\end{abstract}

Palabras clave: Trastornos Mentales; Investigación Cualitativa; Estilo de Vida Saludable; Promoción de la Salud; Bienestar Social.

\section{ASSESSMENT OF THE FACTORS THAT DETERMINE THE ACQUISITION OF HEALTHY EATING HABITS IN PEOPLE WITH SEVERE MENTAL DISORDER: QUALITATIVE APPROACH FROM A FIRST- PERSON PERSPECTIVE AND FROM PROFESSIONALS}

\begin{abstract}
Introduction: Adoption unhealthy diets is related to the appearance of metabolic alterations a contributing factor to the high rates of comorbidities in people with severe mental illness. Knowing the determining factors allows to develop food education programs appropriate to the needs of the person; Goals: To explore the factors that determine the adoption and maintenance of eating habits, and identify the most efficient strategies for changing habits; Methods: Descriptive study with qualitative data collection and analysis. Data was obtained from 6 discussion groups. A thematic analysis of the corpus was carried out; Results: 23 people attended to mental health services and 30 professionals from different care areas participated. Six main themes emerge; Conclusions: Professionals and users agree on the need to promote healthy eating. Knowing the predisposition and conditioning factors to adopt a behaviour change is essential for the design of efficient interventions.
\end{abstract}

Keywords: Mental Disorders; Qualitative Research; Healthy Lifestyle; Health Promotion; Social Welfare.

\section{INTRODUCCIÓN}

Las personas con TMG presentan una tasa de mortalidad media de 2 a 3 veces superior a la de la población general, reduciendo la esperanza de vida entre 10 y 25 años (Liu et al., 
2017)回. En este contexto, la promoción, prevención y tratamiento de la salud de las personas con trastorno mental se considera una línea prioritaria de intervención desde el punto de vista de la salud pública (Departament de Salut de la Generalitat de Catalunya, 2017)?.

La elevada mortalidad presenta una causa multifactorial, incluyendo factores de riesgo individual, asociados a la enfermedad o en la adopción de comportamientos específicos, junto con factores de riesgo asociados al sistema sanitario y a determinantes socioambientales. Los factores de riesgo individual incluyen características inherentes al trastorno y a los comportamientos relacionados con la salud de la persona. Dichos factores se pueden atribuir a la fisiopatología, la severidad del trastorno y a la sintomatología asociada, junto con la adopción de estilos de vida poco saludables (Liu et al., 2017)]

La elevada morbilidad y mortalidad en muchos casos se debe a la aparición de patologías concurrentes, siendo especialmente prevalentes las patologías cardiovasculares. Esta condición es debida a la elevada incidencia de alteraciones metabólicas, relacionadas con una serie de factores de riesgo cardiovascular, como la obesidad, la hipertensión, la diabetes mellitus tipo 2 y la síndrome metabólica (Ayerbe et al., 2018)]. La presencia de alteraciones metabólicas, especialmente la obesidad, se relaciona a su vez con una disminución del nivel de funcionalidad y un empobrecimiento de la calidad de vida de la persona (Cerimele \& Katon, 2013)国.

Es sabido que las personas con TMG tienden a adoptar hábitos alimentarios desequilibrados desde una perspectiva dietética-nutricional, resultando en un patrón alimentario definido por el seguimiento de dietas hipercalóricos, junto a una baja presencia de alimentos de origen vegetal (Jahrami, Faris, Saif, \& Hammad, 2017)]? Los objetivos del abordaje dietético están enfocados a promover una alimentación saludable y adecuada a las necesidades individuales como parte fundamental del tratamiento integral de la persona, para prevenir y controlar las comorbilidades asociadas, junto a la promoción del bienestar psicológico y la satisfacción (Kaur, Bhatia, \& Gautam, 2014)国.

Distintos factores influyen en la toma de decisiones y condicionan las prácticas alimentarias adoptadas (Aschbrenner et al., 2013)], siendo en cierta medida modificables mediante programas de educación alimentaria. Entender a las personas, sus comportamientos alimentarios y el contexto en el que se desenvuelven es crucial para desarrollar 
intervenciones con el objetivo de adecuar las prácticas y elecciones alimentarias (Contento, 2011)回.

La mayoría de las intervenciones desarrolladas para incidir en los estilos de vida de las personas con TMG se centran en la reducción del peso corporal o en el incremento de la actividad física, para reducir la incidencia de alteraciones metabólicas y consecuentemente mejorar el estado de salud (Naslund et al., 2017)]. Son pocas las intervenciones que muestran resultados favorables sostenidos en el tiempo (Holt et al., 2019)], hecho atribuible a las limitaciones metodológicas de las intervenciones 0 a la inadecuación de los contenidos. Es por este motivo que se considera primordial explorar las necesidades de la población y conocer aquellos elementos que condicionan la adquisición de unos hábitos alimentarios saludables en vistas a desarrollar estrategias de intervención eficientes y sostenibles.

\section{METODOLOGÍA}

El presente estudio se enmarca dentro de un estudio más amplio que tiene por objetivo evaluar la eficacia de un programa de educación alimentaria basado en el apoderamiento de las personas con TMG para la promoción del consumo de fruta y verdura (DIETMENT), en el que se desarrolla una estrategia de educación alimentaria específica.

Los objetivos se centrar en conocer y contrastar las opiniones de los profesionales y usuarios de los servicios de salud mental en relación a los factores que condicionan la adopción y mantenimiento de los hábitos alimentarios en personas con TMG, e identificar las estrategias más eficientes para el cambio de hábitos.

Estudio descriptivo mediante la recogida de datos y análisis cualitativo, en el que se han realizado 6 grupos de discusión con las personas con diagnóstico clínico de TMG vinculadas activamente a Osonament - Fundació Centre Mèdic Psicopedagògic d'Osona (3 grupos), servicios de atención integral a personas con problemas de salud mental y adicciones de la comarca de Osona (Barcelona, Cataluña), junto con los profesionales de los dispositivos de salud mental de la comarca de Osona que atienden a las personas con TMG (3 grupos) a nivel social y sanitario.

Los grupos se condujeron mediante dos guiones semiestructurados a partir de 5 preguntas abiertas que se discutieron durante 40 a 80 minutos. Una vez obtenida la información de 
ambos colectivos, se realizó la transcripción literal de las diferentes dinámicas grupales. Se realizaron varias lecturas para la familiarización con la información obtenida y empezar a generar los códigos iniciales mediante un proceso de codificación mixta, con el objetivo de focalizar, simplificar y abstraer los datos relevantes agrupándolos en categorías temáticas (Braun \& Clarke, 2006).

Se optó por analizar los datos a través del análisis temático (Boyatzis, 1998)] con el soporte del programa Atlas.ti 8. Se identificó un total 6 temas o categorías principales con diversas subcategorías.

El proceso de codificación fue realizado independientemente por dos investigadores y posteriormente contrastado con los participantes.

Todos los participantes participaron voluntariamente en el estudio. Los datos fueron tratados confidencialmente, respetando en todo momento los derechos de la persona.

\section{RESULTADOS}

Se realizaron 3 grupos de discusión con los usuarios de los servicios de salud mental y 3 con los profesionales de atención, con una participación total de 23 y 30 personas respectivamente. El perfil de los participantes se describe en las Tablas 1 y 2.

Tabla 1. Descripción del perfil de la muestra referente a los usuarios.

\begin{tabular}{|c|c|c|c|c|}
\hline & Grupo A & Grupo B & Grupo C & Total \\
\hline Número participantes & 9 & 8 & 6 & 23 \\
\hline Edad (años) & $50,2(\mathrm{DE} 7,8)$ & 49,7 (DE 13,1) & 43,3 (DE 4,3) & 48,3 (DE 9,4) \\
\hline Sexo & $22,2 \%$ Hombre & $62,5 \%$ Hombre & $100 \%$ Hombre & $56,5 \%$ Hombre \\
\hline $\begin{array}{r}\text { Trastorno depresivo } \\
\text { Trastorno bipolar } \\
\text { Trastorno de personalidad } \\
\text { Espectro esquizofrenia y } \\
\text { otros trastornos psicóticos }\end{array}$ & \begin{tabular}{|l|}
$22,2 \%$ \\
$11,1 \%$ \\
$0 \%$ \\
$66,7 \%$
\end{tabular} & \begin{tabular}{|l|}
$25 \%$ \\
$25 \%$ \\
$12,5 \%$ \\
$37,5 \%$ \\
\end{tabular} & \begin{tabular}{|l|}
$16,7 \%$ \\
$0 \%$ \\
$16,7 \%$ \\
$66,7 \%$
\end{tabular} & \begin{tabular}{|l|}
$21,7 \%$ \\
$13 \%$ \\
$8,7 \%$ \\
$56,5 \%$
\end{tabular} \\
\hline Años del diagnostico & 10,9 & 11,1 & 11,7 & 11,2 \\
\hline
\end{tabular}


Tabla 2. Descripción del perfil de la muestra referente a los profesionales.

\begin{tabular}{|c|c|c|c|c|}
\hline & Grupo A & Grupo B & Grupo C & Total \\
\hline Número participantes & 11 & 7 & 12 & 30 \\
\hline Edad (años) & 36 (DE 6,0) & 37,4 (DE 5,0) & 30,2 (DE 9,4) & 34 (DE 7,8) \\
\hline Sexo & $18,1 \%$ Hombre & $14,2 \%$ Hombre & $25 \%$ Hombre & $20 \%$ Hombre \\
\hline $\begin{array}{r}\text { Psicólogo } \\
\text { Terapeuta ocupacional } \\
\text { Educador social } \\
\text { Trabajador social } \\
\text { Integrador social } \\
\text { Enfermero } \\
\text { Auxiliar de enfermería } \\
\text { Psiquiatra } \\
\text { Monitor } \\
\text { Estudiante en prácticas } \\
\text { Técnico Actividad Física }\end{array}$ & $\begin{array}{l}9,1 \% \\
36,4 \% \\
18,2 \% \\
0 \% \\
27,3 \% \\
0 \% \\
0 \% \\
0 \% \\
0 \% \\
0 \% \\
9,1 \% \\
\end{array}$ & $\begin{array}{l}28,6 \% \\
14,3 \% \\
42,9 \% \\
0 \% \\
0 \% \\
0 \% \\
0 \% \\
0 \% \\
14,3 \% \\
0 \% \\
0 \% \\
\end{array}$ & \begin{tabular}{|l|}
$16,7 \%$ \\
$0 \%$ \\
$0 \%$ \\
$8,3 \%$ \\
$0 \%$ \\
$25 \%$ \\
$8,3 \%$ \\
$16,7 \%$ \\
$8,3 \%$ \\
$16,7 \%$ \\
$0 \%$ \\
\end{tabular} & \begin{tabular}{|l|}
$16,7 \%$ \\
$16,7 \%$ \\
$16,7 \%$ \\
$3,3 \%$ \\
$10 \%$ \\
$10 \%$ \\
$3,3 \%$ \\
$6,7 \%$ \\
$6,7 \%$ \\
$6,7 \%$ \\
$3,3 \%$ \\
\end{tabular} \\
\hline $\begin{array}{l}\text { Años experiencia } \\
\text { profesional }\end{array}$ & 11,4 & 12,1 & 4,7 & 8,9 \\
\hline
\end{tabular}

El análisis temático de la información se presenta en relación a la comparación de las opiniones emergidas entre los dos colectivos (usuarios y profesionales) para explorar puntos coincidentes y divergentes entre ellos. A continuación se presentan los 6 temas principales obtenidos:

\subsection{Tema 1. Conocimientos sobre alimentación saludable frente a las prácticas alimentarias}

En general los usuarios son conocedores de los aspectos más básicos en relación a la alimentación saludable. Algunas personas tienen conocimientos más específicos, hablan de nutrientes y raciones de consumo de determinados alimentos. Sin embargo, aparecen dudas en relación a cómo seguir una alimentación saludable y se identifican algunos aspectos que generan confusión.

Cuando se explora la valoración sobre las prácticas alimentarias, se observa que la mayoría asumen que no comen de forma saludable, pese a saber identificar los aspectos que podrían modificar para adecuar su alimentación. Asimismo, algunas personas tienen una visión distorsionada sobre qué significa una alimentación saludable, asociándola a una dieta restrictiva carente de placer.

Usuario 8 "Debería comer creo un poco más variado, en el sentido de comer algo de carne, que no la pruebo casi, y de comer más fruta y más verdura que los cómo sólo uno o dos o tres veces a la 
semana"; Usuario 17 "No como bien, quiero decir que no tengo horarios, como solo dos veces al día... no considero que este comiendo correctamente"; Usuario 21 "Ya hay pocos placeres en esta vida, sólo falta que me quiten la comida que me gusta"

\subsection{Tema 2. Asociación entre la alimentación saludable y la salud}

Ambos grupos manifiestan los beneficios de seguir un patrón alimentario saludable y destacan las repercusiones de su déficit a nivel de salud física y mental, ya sea a nivel de estado de ánimo ó bienestar personal. Los profesionales apuntan también a su relación con la calidad de vida y a la estabilidad psicopatológica de la persona.

Sin embargo en diversas ocasiones, y desde ambas perspectivas, sólo se relaciona el consumo de alimentos de baja calidad nutricional o la adopción de conductas alimentarias poco saludables a repercusiones vinculadas a la ganancia de peso corporal, y no se atribuye a consecuencias mayores a nivel de salud.

Desde la perspectiva de los profesionales, pese a haber consenso respecto a la importancia que los usuarios sigan una alimentación saludable y en su relevancia como una herramienta más para trabajar la salud mental de la persona, aparecen discrepancias en cuanto a su trabajo: algunos sólo lo consideran un tema prioritario cuando aparece una disfunción o una patología orgánica de base, en cambio otros lo consideran como un aspecto a trabajar en paralelo a diferentes aspectos en el proceso de recuperación de la persona.

Usuario 17 "Yo creo que mejorando esta parte de la alimentación me hará sentir mejor, supongo que si me siento mejor por dentro también me sentiré mejor mentalmente"; Profesional A7 "Yo creo que sí es un pilar, pero no el único ni mucho menos"; Profesional B1 "No tienes el tiempo en las visitas, tienes que trabajar otras cosas aparte de la alimentación"

\subsection{Tema 3. Factores que condicionan positivamente la adquisición de hábitos alimentarios saludables}

Se desprenden dos subcategorías: condicionantes intrínsecos a la persona y condicionantes extrínsecos. Referente a los condicionantes intrínsecos, profesionales y usuarios identifican la autoestima, la motivación, tener mayores conocimientos y ser consciente de la importancia y beneficios de comer de forma saludable, aspectos que hacen más fácil adoptar y mantener los cambios en el tiempo.

Diversos profesionales coinciden que cuando el asesoramiento va precedido de una demanda implica mayor motivación, por contra, cuando el profesional es quien impone el 
cambio mayoritariamente los resultados derivan a intervenciones no efectivas. A su vez, identifican como elemento favorecedor tener una actitud pro-activa y una mayor implicación en el proceso de cambio y toma de decisiones.

Profesional A13 "Yo creo que es más importante la motivación, porque puede haber conciencia pero... decir ya sé que lo tengo que hacer, pero me da igual"; Profesional A8 "La persona que ha tenido predisposición para el cambio, yo tengo algunos casos concretos y se ha notado muchísimo"

En relación a los condicionantes extrínsecos, son pocos los puntos comunes, aun así, ambos grupos perciben el apoyo social como un aspecto favorecedor, siempre y cuando el entorno siga unos hábitos alimentarios saludables o esté dispuesto a adoptar cambios conjuntos. Los profesionales también apuntan a un entorno que fomente unos hábitos saludables, ya sea a nivel de entidad como de círculo familiar o social, que haga más accesible el consumo de alimentos saludables, tanto a nivel físico como económico. A su vez, los usuarios valoran positivamente contar con alguien que se responsabilice de la alimentación, sobre todo en aquellos momentos donde la astenia, desmotivación o estados anímicos más bajos están más presentes.

Usuario 13 "Yo creo que hago una dieta equilibrada porque no soy yo quien la hace, quiero decir es mi madre quien organiza la alimentación"; Usuario 15 "También hay quien cocina y hay a quien le hacen la comida, aquí la diferencia es muy importante, si te lo tienes que hacer tú el estado de ánimo puede influir, te puedes descuidar"

Los profesionales sugerían realizar cambios progresivos de acuerdo con la predisposición de la persona, evitando cambios muy drásticos en su funcionamiento habitual. Enfatizaban en la persistencia e insistencia de los mensajes que se dan, que sean reiterativos y que se haga un trabajo constante para que la persona pueda ir integrándolo en su día a día. Asociar beneficios sociales al trabajo de la alimentación, dar valor a los beneficios de seguir una alimentación saludable, compartir el proceso con otras personas y objetivar los cambios con medidas de impacto o indicadores de salud se describieron como elementos que favorecen la motivación de la persona y fomentan el trabajo del hábito, facilitando consecuentemente su adopción.

Profesional A9 "No hacer grandes cambios, partiendo de su rutina habitual mirar que se puede añadir, quitar o cambiar"; Profesional B2 "Yo considero que sería importante asociar beneficios, es decir, después de hacer una buena comida y hacerlo con compañía de alguien, tener la posibilidad de tener una conversación, asociar beneficios de carácter más social" 


\subsection{Tema 4. Factores que condicionan negativamente la adquisición de hábitos alimentarios saludables}

Se identificaron diversas barreras para la adopción y mantenimiento de una hábitos alimentarios saludables organizadas en dos subcategorías: los condicionantes intrínsecos a la persona y los condicionantes extrínsecos. En relación a los condicionantes intrínsecos, profesionales y usuarios destacan el estado emocional, acentuando la ansiedad y las situaciones de angustia. Estas situaciones pueden derivar a "atracones" o, por el contrario, a la privación de las ingestas, sesgando la percepción de hambre respecto a la distinción del estado, a la vez que también pueden condicionar la toma de decisiones alimentarias. A nivel de los usuarios, en algún caso, se verbaliza la falta de motivación para cambiar la forma de alimentarse, ya sea por falta de interés o bien por pereza. Ambos grupos identifican factores inherentes al trastorno. La estabilidad clínica, la sintomatología negativa, la rigidez o el deterioro cognitivo pueden dificultar el mantener o adoptar estilos de vida saludables.

Profesional A17 "Los estados de ánimo a veces si que determinan, cuando hay estados más ansiosos o
más depresivos condiciona si compras más alimentos hipercalóricos, o si tienes más apetencia por el
dulce"; Usuario 17 "Yo me estoy dando cuenta que me estoy haciendo mayor y que no estoy saludable,
pero por cuestión de ánimos tampoco hago nada, es como un pez que se come la cola"; Profesional A1
"Pensar que ellos tal vez antes de los trastornos ya tenían un hábitos saludables, pero quizás el propio
deterioro o el estado anímico bajo hace que ahora la persona se descuide, incluso en lo que tenía más
integrado" En diversas ocasiones, ambos grupos mencionan las preferencias y aversiones alimentarias. Los usuarios remarcan la aversión hacia la fruta y la verdura, lo que comporta un consumo insuficiente. Los profesionales apuntan también a una mayor preferencia hacia las técnicas culinarias menos recomendadas y hacia alimentos de baja calidad nutricional. Por otro lado, la falta de habilidades o la falta de interés en la cocina limita las opciones a las comidas preparadas.

Usuario 8 "Yo no puedo hacer dieta, porque si me dices que no puedo comer lo que me gusta, por ejemplo la bollería y este tipo de cosas, luego me entran más ganas de comerlo"; Usuario 21 "No me gustan demasiadas cosas, las patatas fritas y la carne, los vegetales no los como"

En relación a los atributos externos, ambos grupos identifican el factor económico. Asimismo, los usuarios relacionan el hecho de no disponer de apoyo social a repercusiones en la calidad de la alimentación, ya sea por el tipo de alimento que se consume o bien por la cantidad. Los profesionales apuntan a que aquellas personas que viven solas o bien no están acompañadas en las comidas tienden a descuidar su alimentación. Por el contrario, 
ambos coinciden que no tener el liderazgo en la toma de decisiones alimentarias o en la preparación de las comidas puede condicionar negativamente el cambio de hábitos. Siempre que las personas que se responsabilizan de la alimentación no sigan un patrón alimentario saludable o se nieguen a adoptar cambios.

Profesional A1 "Algunas personas son conscientes de que deberían comer más producto fresco, pero económicamente no se lo pueden permitir"; Usuario 11 "Se come peor estando solo, se cocina menos. A mí me gusta mucho cocinar para la gente, pero para mí mismo cocino como si cocinara para una mascota"; Profesional A1 "A veces es eso, es la familia la que no tiene un hábitos saludables, es quien prepara los alimentos, ya puedes ir trabajando con la persona que el problema está más atrás y nosotros no tenemos acceso"

Los profesionales atribuyen la fácil accesibilidad a alimentos de bajo valor nutricional junto a la falsa percepción que estos alimentos son más fáciles de preparar, más saciantes y placenteros a nivel gustativo al hecho que sean alimentos de primera elección para muchas personas.

El tratamiento farmacológico y los consiguientes efectos secundarios, también se identificaron como factores que interfieren en la adopción de los hábitos alimentarios saludables, aunque dicha relación solo es mencionada por los profesionales.

\subsection{Tema 5. Estrategias educativas eficientes}

Se identificó que la alimentación es un tema poco trabajado. Aun así las personas con TMG están interesadas en participar de actividades focalizadas a promocionar la alimentación saludable.

Ambos grupos destacaron que sería más favorecedor abordar la alimentación desde un enfoque grupal. Los usuarios valoraron positivamente disponer de un espacio donde poder compartir experiencias y opiniones. A su vez, se apuntaba que aquellas personas conocedoras o expertas en algún tema dietético pudieran transmitirlo a otros, dándole un formato de grupo de iguales. Todos los profesionales coincidieron en que la intervención grupal es muy positiva, tanto por el refuerzo obtenido a través del grupo, como por devenir en una fuente de motivación. Ambos colectivos también subrayaron el hecho de complementar el trabajo grupal con asesoramientos y seguimientos individuales, para reforzar la parte de planificación de menús y adaptar la intervención y el plan de trabajo a las necesidades y oportunidades de la persona. 
Profesional A4 "La parte esta grupal es importante, porque tal vez por mi solo me cueste pero en grupo veo que hay otras personas que me pueden ayudar en esto, y compartimos"; Usuario 17 "Tener un menú me iba muy bien, porque hacia las cinco comidas, desayunaba por la mañana y todo esto me iba bien, no sé si perdí peso o no, pero me fue bien hasta que lo terminé, luego se acabó”

Se comparte la visión de basar las intervenciones en metodologías vivenciales, trabajarlo desde la vertiente práctica, para que sea más fácil de trasladar y aplicar a las rutinas diarias, hecho que aumenta la motivación y facilita la adherencia al cambio. Algunos aspectos que se desprenden son: soporte en la planificación de la alimentación y en la compra, prácticas y cursos de cocina, o alternativas saludables a los alimentos de consumo habitual.

Profesional B2 "Una intervención mucho más práctica, mucho más vivencial por el paciente, que la persona vea directamente el trabajo, lo manipule, ve los beneficios de lo que está haciendo, que una idea o un concepto mucho más teórico que quizá después le cueste integrar esta información y no le ve la utilidad"

\subsection{Tema 6. Complejidad en el abordaje de la alimentación}

En reiteradas ocasiones los usuarios han manifestado la dificultad para seguir una alimentación saludable, algunos de los motivos que se aducen son la no inmediatez de los beneficios, junto con la complejidad en la sostenibilidad de los cambios, sobre todo cuando se desdibuja la figura del profesional, lo que conlleva al abandono de la conducta adquirida antes de evidenciar resultados.

La dificultad no sólo es percibida por parte de las personas atendidas, sino también por el propio profesional. Atribuyen dicha dificultad a la falta de conocimientos o a la carencia de herramientas y estrategias para su abordaje, junto con la falta de tiempo para trabajarlo. Se describieron distintos modelos de atención profesional donde se interviene en la alimentación, aunque siempre constituyendo un apoyo muy superficial.

Los profesionales plantean la necesidad de establecer un referente en alimentación, mediante la figura del dietista-nutricionista, para poder ofrecer un asesoramiento adecuado y resolver las demandas que puedan derivarse. También, se consideró relevante aumentar la evidencia científica basada en resultados contrastados para fomentar la necesidad de intervención en la alimentación de las personas con TMG.

Usuario 19 "La dieta en sí quizás no sea difícil, pero lo que es difícil es mantenerla, mantenerla es lo que es más difícil pienso yo"; Profesional A2 "Siempre somos los mismos diciendo que deben vigilar con el 
azúcar, que al final no le sabes ni explicar lo que es la diabetes, que haya un profesional que se lo explique, que le haga el seguimiento, esto es lo que motiva"

\section{DISCUSIÓN Y CONCLUSIONES}

Los resultados principales se focalizan en conocer y contrastar las opiniones en relación a la promoción de la alimentación en los servicios de salud mental, desde la perspectiva de las personas atendidas y de los profesionales de distintos ámbitos de atención. En general aparecen pocas discrepancias entre ambos grupos, cada uno aporta una visión complementaria a la del orto, resultando en un conocimiento más global.

Ambas partes definen un patrón alimentario caracterizado por el seguimiento de dietas desequilibradas, debido a la falta de adecuación a las frecuencias y raciones de consumo de determinados grupos de alimentos, junto con una desestructuración de los horarios de las comidas, tal y como algunos autores ya han descrito anteriormente (Jahrami et al., 2017)? El consumo de alimentos de baja calidad nutricional o la adopción de conductas alimentarias poco saludables se atribuyen principalmente a repercusiones vinculadas al aumento de peso corporal y no a consecuencias mayores a nivel de salud. Aún conociendo los aspectos básicos de la alimentación saludable estos conocimientos no siempre se trasladan a la práctica habitual, prevaleciendo la apetencia hacia determinados tipos de alimentos frente a los atributos vinculados a la salud. Barre, Ferron, Davis y Whitley (2011)] relacionan la no predisposición al cambio a una falta de consciencia, situando a estas personas en una fase pre-contemplativa o contemplativa. A su vez, Yarborough, Stumbo, Yarborough, Young y Green (2016)] apuntan que el cambio de hábitos relacionado a los estilos de vida está motivado principalmente por la percepción del riesgo a eventos adversos relacionados con la salud.

Asimismo, los usuarios perciben la alimentación saludable como un tema complejo debido a las dificultades para su adopción y mantenimiento. Ambas partes identifican diversos aspectos percibidos como barreras en el cambio de hábitos, relacionados con la conducta y capacidades de la persona, a factores inherentes al trastorno, junto con atributos externos vinculados con el entorno social y comunitario. Otros autores también señalan al rol de ciertos alimentos como fuente de placer y su contribución al bienestar emocional de la persona o a la priorización de la salud mental frente a la alimentación saludable (Barre et al., 2011)国. Para implementar intervenciones eficientes y sostenibles dirigidas a adecuar los hábitos alimentarios de las personas con TMG, es fundamental conocer y tener en 
consideración los condicionantes negativos, e interferir sobre aquellos que se consideran modificables. Asimismo, cabe destacar e incluir los factores que facilitan la predisposición al cambio, ya sea desde una perspectiva intrínseca o extrínseca a la persona (McKibbin, Kitchen, Wykes, \& Lee, 2014).

Enfocar las estrategias de educación alimentaria sobre actividades grupales basadas en metodologías prácticas y vivenciales, junto con su combinación a un asesoramiento individualizado, fue un punto común entre profesionales y usuarios. Se resalta basar las intervenciones a la demanda y estímulo de la persona, hecho que acaba reportando mayor motivación y resultados más favorables con respecto a la adopción y adherencia al cambio de hábitos (Graham et al., 2014).

La complejidad en el abordaje de la alimentación no solo es percibida por parte de los usuarios, los profesionales manifiestan tener pocos conocimientos, recursos y estrategias de intervención para hacer frente a las demandas. Aunque se trabaje desde distintos servicios y áreas de atención, siempre es de una manera muy superficial. Asimismo, se plantea la profesionalización en el abordaje de la alimentación, mediante la figura del dietistanutricionista para poder ofrecer un asesoramiento adecuado y dar respuesta a las necesidades de las personas. Del mismo modo, en el estudio de Mahony, Haracz y Williams (2012), los profesionales de atención manifestaron tener limitaciones en el asesoramiento nutricional.

Se insiste en la necesidad de realizar más estudios que permitan explorar las opiniones de las personas con TMG en relación al abordaje de la alimentación y las estrategias más adecuadas para su promoción, permitiendo conocer sus necesidades reales, junto con los condicionantes para su adopción. Focalizar la intervención hacia la predisposición al cambio de la persona permite diseñar estrategias metodológicamente más adecuadas, eficientes y sostenibles, reportando resultados más favorables y adecuando la gestión de los recursos profesionales.

\section{REFERENCIAS}

Aschbrenner, K., Carpenter-Song, E., Mueser, K., Kinney, A., Pratt, S., \& Bartels, S. (2013). A Qualitative Study of Social Facilitators and Barriers to Health Behavior Change Among Persons with Serious Mental IIIness. Community Ment Health J, 49, 207-212. 
Ayerbe, L., Forgnone, I., Foguet-Boreu, Q., González, E., Addo, J., \& Ayis, S. (2018). Disparities in the management of cardiovascular risk factors in patients with psychiatric disorders: A systematic review and meta-analysis. Psychological Medicine, 48(16), 2693-2701.

Barre, L. K., Ferron, J. C., Davis, K. E., \& Whitley, R. (2011). Healthy eating in persons with serious mental illnesses: Understanding and barriers. Psychiatric Rehabilitation Journal, 34(4), 304-310.

Boyatzis, R. (1998). Transforming qualitative information: Thematic analysis and code development. Thousand Oaks, CA: Sage.

Braun, V., \& Clarke, V. (2006). Using thematic analysis in psychology. Qualitative Research in Psychology, 3(2), 77-101.

Cerimele, J. M., \& Katon, W. J. (2013). Associations between health risk behaviors and symptoms of schizophrenia and bipolar disorder: A systematic review. Gen Hosp Psychiatry, 35(1), 16-22.

Contento, I. R. (2011). Overview of determinants of food choice and dietary change: Implications for nutrition education.Conte. En Nutrition Education: Linking Research, Theory and Practice. 2 ed. Jones and Bartlett Publishers.

Departament de Salut de la Generalitat de Catalunya. (2017). Pla integral d'atenció a les persones amb transtorn mental i addicions. En Generalitat de Catalunya.

Graham, C., Rollings, C., de Leeuw, S., Anderson, L., Griffiths, B., \& Long, N. (2014). A qualitative study exploring facilitators for improved health behaviors and health behavior programs: mental health service users' perspectives. The Scientific World Journal, 2014, 870497.

Holt, R. I. G., Gossage-worrall, R., Hind, D., Bradburn, M. J., Mccrone, P., Morris, T., ... Wright, S. (2019). Structured lifestyle education for people with schizophrenia, schizoaffective disorder and first-episode psychosis (STEPWISE): randomised controlled trial. The British Journal of Psychiatry, 214, 63-73.

Jahrami, H. A., Faris, M. A. I. E., Saif, Z. Q., \& Hammad, L. H. (2017). Assessing dietary and lifestyle risk factors and their associations with disease comorbidities among patients with schizophrenia: A case-control study from Bahrain. Asian Journal of Psychiatry, 28, 115-123.

Kaur, J., Bhatia, M. S., \& Gautam, P. (2014). Role of dietary factors in Psychiatry. Delhi Psychiatry Journal, 17, 452-457.

Liu, N. H., Daumit, G. L., Dua, T., Aquila, R., Charlson, F., Cuijpers, P., ... Saxena, S. (2017). Excess mortality in persons with severe mental disorders: a multilevel intervention framework and priorities for clinical practice, policy and research agendas. World Psychiatry, 16, 30-40.

Mahony, G., Haracz, K., \& Williams, L. T. (2012). How mental health occupational therapists address issues of diet with their clients: a qualitative study. Australian Occupational Therapy Journal, 59, 294-301.

McKibbin, C. L., Kitchen, K. A., Wykes, T. L., \& Lee, A. A. (2014). Barriers and facilitators of healthy lifestyle among persons with serious and persistent mental illness: Perspectives of community mental health providers. Community Ment Health J, 50(5), 566-576.

Naslund, J. A., Whiteman, K. L., Mchugo, G. J., Aschbrenner, K. A., Marsch, L. A., \& Bartels, S. J. (2017). Lifestyle interventions for weight loss among overweight and obese adults with serious mental illness: A systematic review and meta-analysis. Gen Hosp Psychiatry, 47, 83-102.

Yarborough, B. J. H., Stumbo, S. P., Yarborough, M. T., Young, T. J., \& Green, C. A. (2016). Improving lifestyle interventions for people with serious mental illnesses: Qualitative results from the STRIDE study. Psychiatr Rehabil J, 39(1), 33-41. 